\title{
Expanded Disability Status Scale
}

National Cancer Institute

\section{Source}

National Cancer Institute. Expanded Disability Status Scale. NCI Thesaurus. Code 198302.

A system for quantifying disability in multiple sclerosis (MS). Based on a standard neurological examination, the Expanded Disability Status Scale quantifies disability in seven Functional Systems (plus "Other") and assigns a Functional System Score in each. Results are ranked as steps from 0 to 10, with steps 1 through 4.5 describing MS patients who are fully ambulatory and steps 5 through 9.5 describing increasing impairment to ambulation. Step 10 refers to death due to MS. 